\title{
Protective role of sulforaphane against phthalate and bisphenol A mixture linked hepatocellular carcinoma: in silico toxicogenomic datamining
}

\author{
Katarina Baralić*, Katarina Živančević, Dragica Jorgovanović, Dragana Javorac, \\ Evica Antonijević, Aleksandra Buha Djordjevic, Marijana Ćurčić, Zorica Bulat, \\ Biljana Antonijević, Danijela Đukić-Ćosić \\ Department of Toxicology „Akademik Danilo Soldatović“, Faculty of Pharmacy, University of Belgrade, \\ Vojvode Stepe 450, 11000 Belgrade, Serbia
}

\section{Introduction}

Phthalates and bisphenol A have been widely used as plasticizers in many consumer products. In addition to acting as endocrine disruptors, recent studies indicate that these substances promote the progression of several types of cancers, including hepatocellular carcinoma (HCC) (Tsai et al., 2015; Weinhouse et al., 2014). The protective effect of many substances on hepatocellular carcinoma has been investigated in recent years. Sulforaphane (SFN), a very potent isothiocyanate isolated from broccoli, was found to be the most potent naturally occurring inducer of phase 2 enzymes, known to lower susceptibility to chemical carcinogenesis (Priya et al., 2011).

By integrating measuring families of cellular molecules with bioinformatics and conventional toxicology, toxicogenomics can be useful for exploring interactions between genes and chemicals in disease causation, as well as for mixture toxicity assessment and generating hypothesis on chemical therapeutic and protective actions (Boverhof and Zacharewski, 2006).

Thus, the aim of this study was to explore the link between the mixture of two phthalates (bis (2- ethylhexyl) phthalate (DEHP) + dibutyl phthalate (DBP)) and bisphenol A (BPA) and HCC development by using toxicogenomic data mining in silico analysis. Furthermore, this study aims to investigate the ability of sulforaphane to elucidate toxic effects of the investigated mixture on the gene level.

\section{Materials and methods}

The Comparative Toxicogenomic Database (CTD; http://ctd. mdibl.org) was used as the main data mining tool in this research. Batch Query and Set Analyser CTD tools were used to retrieve all curated $\mathrm{HCC}$ - gene interactions for the investigated substances, as well as the molecular pathways these genes were involved in. MyVenn CTD tool was used to find the genes common to all the investigated substances. GeneMania prediction server (https://genemania.org) was used for constructing tight network of genes related to the HCC development. GeneCards: the Human Gene Database (https://www.genecards.org) was used for browsing the genomic, transcriptomic, proteomic, genetic, clinical and functional information about the selected genes.

\footnotetext{
* katherinekatabarka@gmail.com
} 


\section{Results and discussion}

SetAnalyser CTD tool revealed that DEHP, DBP and BPA interact with 183, 222 and 480 HCC related-genes, respectively, all of them listed as HCC marker/mechanism genes. Likewise, SFN interacted with 70 HCC-related genes. However, unlike DEHP, DBP and BPA, it was marked as possible therapeutic agent for HCC.

MyVenn CTD tool revealed the common genes for the investigated substances. DEHP, DBP and BPA interacted with 111 common HCC genes, while 40 of them matched with SFN associated genes. Interactions of the three investigated toxic substances with 14 out of the 40 genes matched (ACTB, CASP8, CCNB1, CYP1A2, CYP2E1, DCN, DPYD, EPHX1, IL6, IRS2, MYC, NFE2L2, PGD, SOD2), while SFN interacted with all of these genes in an opposite manner. GeneMania prediction server was used to construct tight network of the 14 highlighted genes, together with 20 related genes. There was a total of 136 links between these genes. More than half of them $(59.51 \%)$ were in coexpression, while $21.45 \%$ were genetic interactions.

Set Analyzer CTD tool (p-value - 0.01) listed 6 enriched pathways these 14 genes were involved in: FoxO signaling pathway, non-alcoholic fatty liver disease, metabolism of xenobiotics by cytochrome P450, chemical carcinogenesis, phase 1 functionalization of compounds, hepatitis B.

GeneCards database was used to further explore the functions of 14 selected genes (Stelzer et al. 2016). DEHP, DBP and BPA decreased the expression of CASP8 mRNA (gene involved in apoptosis and control of the cell growth) and CCNB1 (gene responsible for control of the cell cycle), while SFN acted oppositely. Furthermore, while DEHP, DBP and BPA decreased the expression of genes involved in the metabolism of xenobiotics and chemical carcinogenesis (CYP1A2, CYP2E1, EPHX1), SFN increased their expression. As for the tumor suppressor genes, DEHP, DBP and BPA decreased the expression of DCN mRNA, while SFN increased its expression. Furthermore, DEHP, DBP and BPA activated proto-oncogene MYC, while SFN inhibited its action.

\section{Conclusion}

Our toxicogenomic in silico data mining identified 14 common HCC-related genes for DEHP, DBP and BPA. Sulforaphane interacted with all of these genes in an opposite manner, indicating a possible protective effect that should be confirmed by further in vitro and in vivo studies.

\section{References}

Boverhof, D.R., Zacharewski, T.R., 2006. Toxicogenomics in risk assessment: Applications and needs. Toxicol. Sci. 89, 352-360. Available at: https://doi.org/10.1093/toxsci/kfj018.

Priya, D.K., Gayathri, R., Gunassekaran, G.R., Sakthisekaran, D., 2011. Protective role of SFN against oxidative stress mediated mitochondrial dysfunction induced by benzo (a) pyrene in female Swiss albino mice. Pulm. Pharmacol. Ther. 24(1), 110-117. Available at: https://doi.org/10.1016/j.pupt.2010.09.002.

Stelzer, G., Rosen, R., Plaschkes, I., Zimmerman, S., Twik, M., Fishilevich, S., Iny Stein, T., Nudel, R., Lieder, I., Mazor, Y., Kaplan, S., Dahary, D., Warshawsky, D., Guan-Golan, Y., Kohn, A., Rappaport, N., Safran, M., Lancet D., 2016. The GeneCards Suite: From Gene Data Mining to Disease Genome Sequence Analysis, Curr. Protoc. Bioinformatics 54(1), 1.30.1 - 1.30.33. Available at: https://doi.org/10.1002/cpbi.5.

Tsai, C.F., Hsieh, T.H., Lee, J.N., Hsu, C.Y., Wang, Y.C., Kuo, K.K., Wu, H.L., Chiu, C.C., Tsai, E.M., Kuo, P.L., 2015. Curcumin suppresses phthalate-induced metastasis and the proportion of cancer stem cell (CSC)-like cells via the inhibition of AhR/ERK/SK1 signaling in hepatocellular carcinoma. J. Agric. Food Chem. 63(48), 10388-10398. Available at: https://doi.org/10.1021/acs.jafc.5b04415.

Weinhouse, C., Anderson, O.S., Bergin, I.L., Vandenbergh, D.J., Gyekis, J.P., Dingman, M.A., Yang, J., Dolinoy, D.C., 2014. Dose-dependent incidence of hepatic tumors in adult mice following perinatal exposure to bisphenol A. Environ. Health Perspect. 122(5), 485-491. Available at: https://doi.org/10.1289/ehp.1307449. 